\title{
Introduction to the Minitrack on Analytics in Support of Continuous Knowledge Creation
}

\author{
Ronald Freeze \\ University of Arkansas \\ rfreeze@walton.uark.edu
}

\author{
Rhonda Syler \\ University of Arkansas \\ rsyler@walton.uark.edu
}

The exponential growth of organizational storage has led to a focus on Big Data and the demand for individual's capable of agilely moving between the data flows and extracting both operational information and knowledge from this ever-increasing store of data. These capabilities have been attributed to the role of Data Scientist. With data being the foundation for the development of information and knowledge, this minitrack focuses on both an organizational and pedagogical perspective for the development and recognition of the Data Scientist. The skill set of the Data Scientist has focused on the abilities of programming, business insights and statistical model building. However, with the rapid accumulation of data stores in organizations, the transfer of data to information and knowledge has become more complex and prior techniques must give way to processes that allow continuous knowledge creation. 